\title{
Exploring the origin of high optical absorption in conjugated
}

\section{polymers}

Michelle S. Vezie ${ }^{1}$, Sheridan Few ${ }^{1}$, Iain Meager ${ }^{2}$, Galatia Pieridou ${ }^{3}$, Bernhard Dörling ${ }^{4}$, R. Shahid Ashraf², Alejandro R. Goñi4,5, Hugo Bronstein ${ }^{2,6}$, Iain McCulloch ${ }^{2,7}$, Sophia C. Hayes ${ }^{3}$, Mariano Campoy-Quiles ${ }^{4 *}$ and Jenny Nelson ${ }^{1 *}$

1. Centre for Plastic Electronics and Department of Physics, Imperial College London, Prince Consort Road, London SW7 2AZ, United Kingdom.

2. Centre for Plastic Electronics and Department of Chemistry, Imperial College London, Exhibition Road, London SW7 2AZ, United Kingdom.

3. Department of Chemistry, University of Cyprus, P. O. Box 20537, 1678 Nicosia, Cyprus

4. Institute of Material Science of Barcelona (ICMAB-CSIC), Campus UAB, 08193, Bellaterra, Spain

5. ICREA, Passeig Lluís Companys 23, 08010 Barcelona, Spain

6. Department of Chemistry, University College London, 20 Gordon Street, London WC1H 0AJ, U.K.

7. SPERC, King Abdullah University of Science and Technology, Thuwal, 23955-6900, Saudi Arabia

\section{ABSTRACT:}

The specific optical absorption of an organic semiconductor is critical to the performance of organic optoelectronic devices. For example, higher light-harvesting efficiency can lead to higher photocurrent in solar cells that are limited by sub-optimal electrical transport. Here, we compared over 40 conjugated polymers, and found that many different chemical structures share an apparent maximum in their extinction coefficients. However, a diketopyrrolopyrrolethienothiophene copolymer shows remarkably high optical absorption at relatively low photon energies. By investigating its backbone structure and conformation with measurements and quantum chemical calculations, we find that the high optical absorption can be explained by the high persistence length of the polymer. Accordingly, we demonstrate high absorption in other polymers with high theoretical persistence length. Visible light harvesting may be enhanced in other conjugated polymers through judicious design of the structure. 


\section{Introduction}

Molecular electronic materials such as conjugated polymers have attracted intense interest for applications in photonics, sensing and solar energy conversion. It is well understood how optical transition energy, optical anisotropy and vibronic broadening relate to the chemical structure of the conjugated backbone and the molecular packing1-5. Several studies report how these properties can be controlled through choice of structure and process route-6-9. Some authors have addressed the broadening of spectral response using panchromatic absorbers ${ }^{10}$ or ternary systems ${ }^{11}$. Absorption spectra have been analysed in terms of the relationship between spectral shape and chemical structure or conformation ${ }^{12-14}$, and individual molecules ${ }^{15}$ or monomers ${ }^{16}$ with high optical extinction have been presented. However, the magnitude of the optical absorption in conjugated polymers has been less well studied and is seldom identified as a design target. The ability to tune the magnitude of absorption could strongly impact applications, for example, by enabling higher photocurrent generation in photodetectors or solar cells with imperfect charge collection, by increasing the radiative efficiency of solar cells ${ }^{17}$ or by increasing the luminance from light emitting diodes.

Figure 1 illustrates the remarkable uniformity of extinction coefficient across a wide range of conjugated polymers, as measured using spectroscopic ellipsometry ${ }^{18}$. Polymers of different chemical structure, self-organising tendency and optical gap lead to a maximum value of $\kappa$ of $0.9 \pm 0.1$, where the complex refractive index $\eta=n_{\mathrm{r}}+i \kappa$. Expressed in terms of the imaginary part of the dielectric function, this maximum lies around $3.9 \pm 0.2$ (corresponding to a linear absorption coefficient of $1.6 \times 10^{5} \mathrm{~cm}^{-1}$ at $700 \mathrm{~nm}$ ). As we show below, this value lies far below their theoretical maximum absorption. Even lower values of $\kappa$ are observed for low bandgap polymers that undergo intrachain charge transfer upon excitation.

In this context, we address the case of the low band-gap polymer, thieno[3,2b] thiophene-diketopyrrolopyrrole (DPP-TT-T). This polymer is interesting on account of the high field-effect transistor mobilities, very promising performance achieved as the donor in 
solar cells, ${ }^{19}$ and high photostability ${ }^{20}$. Moreover the solar cell performance using this polymer has been correlated with the position of the branching point on the polymer side chains ${ }^{21}$ and with the molecular weight of the polymer ${ }^{22}$ but without any convincing mechanism for the trends. Here, we set out to establish the impact of these structural parameters of the polymer on its optical absorption.

\section{Results}

From a set of polymer batches of varying molecular weight (MW) and side chain structure (Tables S1.1 and S1.2) we select four samples for detailed study: high and low molecular weight fractions of the polymer with dodecyl-octyl side chains branched at the second carbon (C1, $M_{n}=120$ and $\left.55 \mathrm{kDa}\right)$ and that with tetradecyl-octyl chains branches at the fourth carbon (C3, $M_{n}=84$ and $16 \mathrm{kDa}$ ) (see Figure $2(\mathrm{a}, \mathrm{b})$ for structures, full molecular weight information is provided in SI Section S1). When applied as the donor component in polymer: $\mathrm{PC}_{70} \mathrm{BM}$ solar cells of device structure glass/Indium tin oxide/ $\mathrm{ZnO} /$ blend(1:2) $/ \mathrm{MoO}_{3} / \mathrm{Ag}$, (active layer thicknesses $\sim 70-100 \mathrm{~nm}$ ) the higher $\mathrm{MW}$ polymers resulted in a substantially larger short circuit photocurrent density, $J_{s c}$, leading to higher power conversion efficiencies of $8.1 \%$ and $8.5 \%$ for C1 HMW and C3 HMW, respectively, compared to the lower MW polymers (5.8\% and 4.6\% for C1 LMW and C3 LMW, respectively). In contrast the effect of the branching point on $J_{\mathrm{sc}}$ for polymers of similar MW is less significant (see inset of Figure 2 (e,f), and SI Section S2). A previous study reporting an effect of branching point on device performance had not resolved molecular weight from side chain structure ${ }^{21}$.

In principle the higher $J_{\mathrm{sc}}$ for the high MW fractions could result from improved electrical properties leading to higher collection efficiency; in the present case, however, the effect cannot readily be explained by active layer thicknesses nor by differences in the charge carrier mobility or lifetime, as measured by charge extraction and transient photovoltage. Whilst mobilities are higher for higher MW polymers (Fig S3.1) consistent with some previous reports ${ }^{23,24}$ the mobility-lifetime products are similar for devices made from different MW 
fractions of either polymer (Figure S3.1). An alternative explanation for the observed changes in $J_{\text {sc }}$ could be differences in optical absorption. We measured the complex dielectric function of the polymers and the corresponding blends with $\mathrm{PC}_{70} \mathrm{BM}$ using variable angle spectroscopic ellipsometry. Figure 2 shows spectra for $n_{\mathrm{r}}$ and $\kappa$ for pristine films and blend films for several molecular weight fractions of $\mathrm{C} 1$ and $\mathrm{C} 3$. The highest molecular weight samples show a $\kappa$ value of about 1.4 (corresponding to an absorption coefficient of $\sim 2.5 \times 10^{5} \mathrm{~cm}^{-1}$ at $700 \mathrm{~nm}$ ), while the lower molecular weight fractions exhibit a maximum $\kappa$ of about 1 , similar to the polymers in Figure 1. Note that all the samples have molecular weights in the range commonly used in organic electronics. For each material, results were confirmed using samples of different film thicknesses, different substrates, and using different ellipsometers. The trend in extinction coefficient of pristine polymer films was reproduced in measurements of blend films (Figure 2 $(\mathrm{e}, \mathrm{f}))$. To establish the contributions of electrical collection efficiency and optical absorption to the observed increase in $J_{\text {sc }}$ we estimate the internal quantum efficiency (IQE) of representative devices using external quantum efficiency (EQE) measurements (Figure S2.2) and a transfer matrix model based on measured optical data for each layer. In each case, enhanced optical absorption is responsible for an increase in $J_{\text {sc }}$ of 8-16\% and improved IQE is responsible for a further, similar increase of 9-32\%. (See Supplementary Information Section S2.3.) This confirms optical extinction as a major cause of higher solar cell performance.

In order to ascertain whether the measured extinction coefficients result from aggregation or anisotropic orientation in the solid state properties, rather than intrinsic properties of the molecules, we measured UV-Vis absorption spectra of dilute solutions of the pure polymers in chloroform and 1,2-dichlorobenzene. The trend in solution is identical to that of films, with the HMW materials absorbing light more strongly at the peak absorption wavelength than the LMW materials (see Fig S5.1). Within the sensitivity of the UV-Vis spectrometer, the pseudo molar extinction coefficient per monomer was unchanged for the range of concentrations studied $(0.25-25 \mu \mathrm{g} / \mathrm{ml}$ in the case of C3) and the spectral shape was 
insensitive to dilution (Fig S5.3). These observations suggest that the absorption phenomena are not the result of chain aggregation in solution; however, we cannot rule out any degree of association between chains.

\section{Discussion}

The results raise two important questions. First, why DPP-TT-T polymers exhibit an optical absorption strength so much higher than the values normally observed for conjugated polymers as shown in Figure 1 and second, how molecular weight affects the magnitude of absorption in this polymer. We address these questions with the help of quantum chemical calculations of the oscillator strength for different materials.

The extinction coefficient $\kappa$ of a molecular material can be related to the molecular orbitals via the transition dipole moment $\mu$ and the oscillator strength $f$. For an optical transition from state $\mid i>$ of energy $E_{\mathrm{i}}$ to state $\mid j>$ of energy $E_{j}$, the transition dipole moment $\mu_{i j}$ is defined as $\boldsymbol{\mu}_{i j}=e\langle j|\hat{r}| i\rangle$ where $\hat{\mathrm{r}}$ is the position operator and $e$ is the electronic charge. The oscillator strength for the transition, assuming that the transition dipoles are oriented at random relative to the direction of the exciting electromagnetic field $\mathcal{E}$, is given by ${ }^{25}$

$$
f_{i j}=\frac{2}{3} \frac{m_{e}}{\hbar^{2} e^{2}}\left(E_{j}-E_{i}\right) \boldsymbol{\mu}_{i j}^{2}
$$

where $m_{\mathrm{e}}$ is the mass of the electron and $\hbar$ is Planck's constant. Note that the sum of oscillator strengths for all possible transitions $i \rightarrow j$ in a system is normalised to the number $N$ of electrons in the system according to the Thomas-Reiche-Kuhn sum rule $\sum_{i, j} f_{i j}=N$.

The linear absorption coefficient $\alpha$ relates to the imaginary part of the complex dielectric function $\varepsilon=\varepsilon_{1}+i \varepsilon_{2}$ through $\alpha=\frac{\omega}{n_{r} c} \varepsilon_{2}$ and also to $\kappa$, via $\alpha=\frac{2 \omega}{c} \kappa$. For a single transition, $\varepsilon_{2}$ can thus be related directly to the transition dipole moment $\mu_{\mathrm{ij}}$ and hence to the oscillator strength. Summing over transitions the spectrum becomes: 


$$
\varepsilon_{2}(\omega)=\frac{2 \pi N_{m} e^{2}}{\varepsilon_{0} m_{e}} \sum_{i, j} \frac{f_{i j}}{\omega} \delta\left(\omega-E_{i j} / \hbar\right)
$$

where $N_{m}$ represents the volume density of species for which $f$ is calculated (e.g. monomers) and the $\delta$ functions can be replaced by functions $D(\omega)$ representing broadened lineshapes. At this stage, we do not resolve each electronic transition into vibronic bands.

To compare the theoretical absorption strength of different conjugated polymers, we use time-dependent density functional theory (TD-DFT) to calculate the oscillator strength and transition energies of the first set of excited state transitions for oligomers of $n=1$ to 8 or more repeat units. We obtain a normalised oscillator strength for the dominant transition, $f_{1}$, in order to compare between oligomer lengths and material systems, by dividing $f_{01}$ (oscillator strength of the first excited state) by the number of $\pi$-electrons in the system, $N_{\mathrm{pi}}$, estimated using Hückel's rule. Most of the oscillator strength in the visible region resides in this first electronic transition; this can be understood in analogy with simple one-dimensional quantum systems such as the harmonic oscillator. (See Supplementary Information, Section S6.1.)

To allow for the effect of chain conformation on optical absorption we consider two limiting cases. For all oligomers studied, the torsional potential between successive monomers has two minima: when successive monomers are rotated by approximately $180^{\circ}$ relative to each other (here referred to as 'all-trans') and when monomers are orientated in the same sense (referred to as 'all-cis'). The 'trans' conformation leads to more linear oligomer structures while 'cis' structures exhibit curvature of the backbone within the conjugated plane. Figure 3(a) shows $f_{1}$ as a function of $N_{\mathrm{pi}}$, calculated for several conjugated oligomers in the linear 'all-trans' conformation. The chemical structures and optimised geometries of the materials and $N_{\text {pi }}$ values are listed in Tables S6.1 and S6.2. In all systems, $f_{1}$ rises with $N_{\text {pi }}$ for small $N_{\text {pi. }}$ Although experimental data on oligomer specific absorption is rare, our results are consistent with experimental measurements of highly monodisperse oligomers of 3-hexylthiophene, which show a rising mass attenuation coefficient in solution with oligomer length up to $N \approx 25$ repeat 
units (see Fig S6.3) ${ }^{26}$; our calculations are also consistent with published data on absorption by polyfluorene 27 and thiophene-co-quinoxelene oligomers ${ }^{28}$. We attribute this rise in $f_{1}$ with $N$ to a superlinear increase in polarisability with oligomer length, as reported for thiophene, acenes, and other elongated conjugated molecules at short lengths ${ }^{29,30}$. In the first excited state $\boldsymbol{\mu}_{01}$ is strongly aligned with the long axis of the oligomer, and capable of coupling strongly with a plane-polarised electromagnetic field.

Both homo-oligomers studied (fluorene and thiophene) in the all-'trans' configuration show larger $f_{1}$ than any donor-acceptor structures, across the calculated range of $N_{\mathrm{pi}}$. This can be attributed in part to their high transition energy relative to the donor-acceptor copolymers (Eq. 1) and doesn't necessarily imply high extinction at any wavelength of interest. In solar cells, for example, we seek high oscillator strength at energies where solar irradiance is high. When the effect of transition energy is removed in Fig. 3(c) by calculating $\varepsilon_{2}$ spectra for the first transition of oligomers of similar size $\left(N_{\mathrm{pi}}=140-150\right)$ in the all-trans conformation the extinction of different materials becomes comparable. Even in this representation DPP-TT-T shows an unremarkable extinction strength. However, when variations in chain conformation are considered, the advantage of DPP-TT-T becomes evident. Fig 3(b) shows $f_{1}$ as a function of $N_{\mathrm{pi}}$ for the same set of materials but in the 'all-cis' configuration when successive monomers are oriented alike and the backbone is curved. Now the specific oscillator strength decreases with $N_{\text {pi }}$ after reaching a maximum. The loss in extinction is due to the oligomer curvature which causes $\mu_{01}$ to increase sublinearly with $N_{\mathrm{pi}}$, but the size of the effect is chemical structuredependent. For example, Si-CPDTBT suffers a strong loss in specific extinction due to its high curvature, resulting from the large angle $\theta_{\text {mon }}$ of $44^{\circ}$ between vectors joining successive monomer pairs while DPP-TT-T with $\theta_{\text {mon }}=27^{\circ}$ and a longer monomer suffers the least (see Figure S6.1). Much of the lost oscillator strength is recovered in higher-lying states, but these are less useful for solar light harvesting. Allowing that at room temperature, any conjugated polymer will sample a range of conformations, the pure 'trans' and pure 'cis' cases represent the 
limits between which the average extinction must lie. In the case of DPP-TT-T the lower (cis) limit lies closer to the upper (trans) limit than for any other polymer studied in this evaluation.

It is important to note that curved and linear oligomers differ in their oscillator strength but not, to a first approximation, in the transition energy since the different conformers studied here are not strained. The effect is captured in the concept of persistence length, which can be related directly to $\mu$, as opposed to conjugation length which is usually related to transition energy ${ }^{31,32}$. DPP-TT-T offers by far the highest theoretical persistence length $\left(\lambda_{\mathrm{p}}\right)$ (of tens of nm, see Fig S6.8) of all materials studied here, as estimated by a simple method adapted from Flory 33, (SI section S6.8) which takes into account the thermodynamic conformational landscape. DPP-TT-T benefits from the relatively long monomer, small $\theta_{\text {mon }}$ and relative preference for 'trans' alignment. The high linearity of DPP-TT-T was also noted in a computational study of polymer conformations in solution ${ }^{34}$. The positive correlation between persistence length and extinction coefficient has been used previously to infer conformation from extinction ${ }^{35}$, but not in the context of designing strongly absorbing conjugated polymers. We note here that calculated values of $\lambda_{\mathrm{p}}$ are generally larger than values determined experimentally 35,36 suggesting that other factors than the theoretical potential energy surface may influence chain extension in practice. We also note that while the relative depth of torsional minima influence $\lambda_{p}$, the steepness of the torsional potential alone is not a critical parameter.

Within this picture we can rationalize a chain length dependence of oscillator strength in DPP-TT-T. In a solution processed polymer sample many conformers will be present in a variety of permutations of relative monomer alignment with chain extension lying between the alltrans and all-cis limits. The estimated persistence length reflects this distribution. The range of conformations together with the monomer length, monomer alignment and torsional potential results in a range of absorption strengths. In the case of DPP-TT-T, the chain curvature and hence oscillator strength is relatively insensitive to chain conformation (i.e. all likely conformations are relatively straight) and this leads to an average extinction that exceeds that 
of all other materials studied here. For completeness, we also analysed the correlation of oscillator strength to spatial overlap of the hole and particle natural transition orbitals and found no correlation (Fig S6.11).

To test the proposal that persistence length dominates optical extinction in solution we identified additional polymers with long monomers and high expected co-linearity (small $\theta_{\text {mon }}$ ), namely, an indacenodithiophene-co-benzothiadiazole polymer IDTBT ${ }^{37}$ and an alternative DPP based polymer diketopyrrolopyrrole-terthiophene (DPP3T). The benefits of the high colinearity of IDTBT are mentioned in Ref ${ }^{38}$. IDTBT and DPP3T each have high $\lambda_{\mathrm{p}}$ and show high solution absorption (Supplementary Figure S9.2). The optical extinction in films of IDTBT and DPP3T reaches a maximum between 1.4 and $1.5\left(\alpha \approx 2.4 \times 10^{5} \mathrm{~cm}^{-1}\right.$ at $\left.700 \mathrm{~nm}\right)$ comparable to DPP-TT-T (Figure 4(a)), and in the case of IDTBT $\kappa$ increases with MW (see Supplementary Figure S9.3). Figure 4(b) illustrates the impact of such an increase in absorption on the external quantum efficiency in a collection limited solar cell, using a simple model and a mobility-lifetime product based on the devices studied here: the increased absorption makes a higher photocurrent available. Much higher electrical quality would reduce the advantage of strong absorption in a solar cell (Fig S10.1a), but practical devices are currently far from that limit.

We now address the MW dependence of the extinction of DPP-TT-T. Examining the gel permeation chromatography data, we find that for both low and high MW fractions, the majority of the MW distributions lie at MWs beyond the point where the calculated specific extinction begins to saturate (Figure S1.1). Therefore the lower specific extinction for low MW polymer is not explained by limited polymer chain length. An alternative hypothesis is that the chains in the LMW and HMW samples are present in different distributions of conformation. This idea is supported by the higher relative strength of the second shoulder (apparent 0-1 vibronic peak) in the absorption spectrum for the LMW than the HMW sample (Fig. 2 for films, Fig S5.1 for solutions). It has been shown that oscillator strength is transferred from the $0-0$ to higher vibronic transitions as a polymer is curved ${ }^{39,40}$. Interestingly, another study also showed that as 
a polymer becomes more coiled oscillator strength is lost from the lowest electronic transition and gained by higher electronic transitions ${ }^{39}$.

To examine the vibronic structure for the DPP-TT-T samples studied here we carried out resonance Raman (RR) spectroscopy on LMW and HMW C3 polymers in solution. The intensities of RR bands are associated with structural changes upon electronic excitation and are thus directly related to the displacement between the ground and excited state potential energy surface minima along specific normal coordinates, determining in turn the shape of absorption spectra. Resonance Raman Intensity Analysis (RRIA) quantifies the Raman spectrum and models the RR cross sections as a function of excitation wavelength for the most intense bands simultaneously with the absorption spectrum, thus providing the most appropriate combination of displacements and transition dipole moments to describe the optical response $\mathrm{e}^{41,42}$.

The resonance Raman spectra for the DPP-TT-T samples and the modeling approach are described in detail in the Supplementary Information, Section S7. Primarily C=C stretching modes either belonging to the TT or the DPP unit are enhanced upon excitation on the blue side of the absorption spectrum. Interestingly, the relative intensities of the bands assigned to TT and DPP units change significantly with excitation wavelength, suggesting contributions from different electronic transitions to the absorption spectrum. Moreover our analysis showed that the RR cross sections and absorption spectra could not be fit simultaneously with a single dipole-allowed electronic transition; such fits greatly overestimated the RR cross sections (Fig. S7.3 and S7.4). However, the RR cross sections were reproduced well when a second electronic transition lying $160 \mathrm{meV}$ above the first was introduced (Fig 5(a, b)), inducing interference between the two transition polarizabilities and thus reducing the RR cross section. The second calculated electronic transition lies at around this energy, $170 \mathrm{meV}$ above the first calculated transition for tetramers of DPP-TT-T. This second calculated transition is dark for linear oligomers but moderately bright in curved (all-cis) oligomers with transition dipole moment $\boldsymbol{\mu}_{02}$ 
oriented perpendicular to the backbone (Fig 5(c)). Therefore the high energy shoulder observed experimentally in the absorption spectrum can be assigned to the sum of two contributions, the second vibronic peak of the first electronic transition and the first vibronic peak of the second electronic transition. Both of these higher energy contributions are expected to be stronger in curved oligomers ${ }^{39,40}$. Our analysis indicates that both electronic transitions and therefore both linear (more 'trans') and curved (more 'cis') conformers exist in both LMW and HMW samples, but the fraction of linear conformers is relatively greater in the HMW case, giving rise to the higher overall oscillator strength. We tentatively assign the higher tendency of chains to adopt linear conformation in the HMW case to the increased strength of chain-chain interactions (which will be maximized for linear chains) over the chain-solvent interactions, consistent with the lower solubility of the longer chains. The hypothesis that chain-chain interactions are more important for higher MW is consistent with the stronger effect of solvent on the absorption spectrum for the LMW than the HMW polymer (Fig. S5.2 and S7.5). The presence of the second electronic transition and conformation dependent distribution of oscillator strength is not unique to DPP-TT-T, and is likely to occur in other materials. RRIA offers a tool to probe such phenomena.

Interestingly, we find little correlation between the push-pull character of the transition and the normalised transition dipole moment of the first transition, $\mu_{01} / N_{\mathrm{pi}}$ for all the polymers we studied (see section S6.10). We also find little charge transfer between the thiophene block and the DPP unit upon excitation to the first excited state of DPP-TT-T, consistent with a previous study ${ }^{20}$, in contrast to the other copolymers studied. We suggest that the low excitation energy in DPP based copolymers is due to coupling of the polymer excitation to the relatively low excitation energy and high oscillator strength of the DPP unit alone (See Fig S6.9 and S6.10). The analysis shows that donor-acceptor character need not restrict the absorption strength of low energy transitions. 
Finally we consider the question of the limit to absorption for a conjugated polymer. Figure 6(a) shows the calculated integral of oscillator strength as a function of photon energy for long oligomers $\left(120<N_{\mathrm{pi}}<165\right)$ of a range of chemical structures. We see that the first transition contributes the majority of oscillator strength in the visible. In every case the integrated $f$ reaches a value much less than unity in the visible, showing that most of the available oscillator strength for the $\pi$-system must reside in higher energy transitions outside the visible range. The low excitation energy and relatively high oscillator strength of DPP-TT-T agree well with the trend shown by the experimental results in Figure 6(b). Also shown in Figure 6(b) are plots of oscillator strength per atom for the conventional semiconductors, silicon, germanium and gallium arsenide. Because the component atoms are, on average, tetravalent in these materials the limiting oscillator strength per atom due to the valence electrons is four. The convergence of the data towards that limit show that these inorganic materials achieve a much higher fraction of their limiting extinction within the visible region than do the organic semiconductors studied.

The examples of DPP-TT-T, DPP3T and IDTBT show that by enhancing the coupling of a conjugated polymer to light through extended persistence length, it can be possible to pull more of the available oscillator strength in to the visible region. Our studies indicate several design considerations to maximise this effect: namely, to target relatively long monomers with high colinearity of successive monomers; to design the torsional potential to be strong and to favour an alternating ('trans') monomer orientation; to achieve low transition energies by using components with low $\pi-\pi^{*}$ excitation energy and high oscillator strength, like the DPP unit, and to exploit the competition between polymer-polymer and polymer-solvent interactions to maximise chain extension in solution. Another strategy would be to reduce the volume fraction taken up by solubilizing side chains, although in practice this may require non solution processing techniques. Whilst the absorption of a polymer in the solid state will also be affected by intermolecular interactions, there is substantial evidence that microstructure in the solid state reflects the structure in solution (e.g. Ref ${ }^{43}$ ). By employing these design considerations, 
our results indicate that polymers can be designed such that their absorption is less sensitive to conformation, thus allowing their full potential to be realised. Exploiting these aspects, along with usual considerations such as charge transport, could open the way to significant improvements in device performance as shown here in the case of solar cells.

\section{Acknowledgements}

M.S.V. and S. F. are grateful to the Engineering and Physical Sciences Research Council (EPSRC) for a doctoral training award and a CDT studentship (EP/G037515/1) respectively. G.P. and S.C.H. acknowledge the University of Cyprus for funding through the internal grant "ORGANIC". B.D., A.G. and M.C.Q. acknowledge financial support from the Ministerio de Economía y Competitividad of Spain through projects CSD2010-00044 (Consolider NANOTHERM), SEV2015_0496 and MAT2012-37776 and the European Research Council through project ERC CoG648901. I.M., R.S.A. and I.McC. acknowledge support from the European Commission FP7 Project ArtESun (604397). J.N. is grateful to the Royal Society for a Wolfson Merit Award, and acknowledges financial support from EPSRC grants EP/K030671/1, EP/K029843/1 and EP/J017361/1. The authors thank Dr. Isabel Alonso for performing supplementary ellipsometric measurements; we thank Prof. Thomas Kirchartz, Dr. Jarvist Moore Frost, Dr. Christian Müller and Dr. Isabel Alonso for helpful discussions.

\section{Author Contributions}

M.S.V. coordinated the experimental work, made films, performed solution UV-Vis measurements, and did electrical characterisation. S.F. did the quantum chemical calculations. I.M. and H.B., supervised by I.McC., made the DPP-TT-T and IDTBT polymers respectively. G.P. and S.C.H. performed the RRS measurements and subsequent analysis. B.D., A.G. and M.C.Q. did the ellipsometry measurements. R.S.A. made the devices. J.N. supervised the work. 
(a)

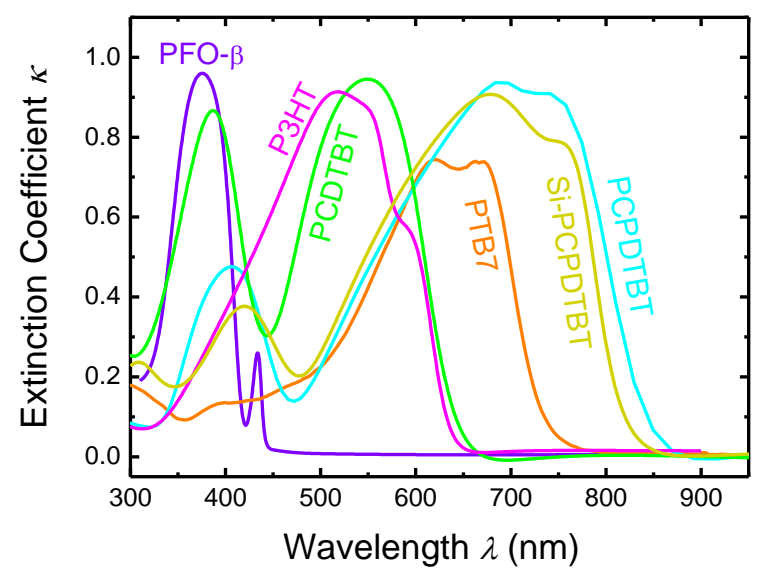

(b)

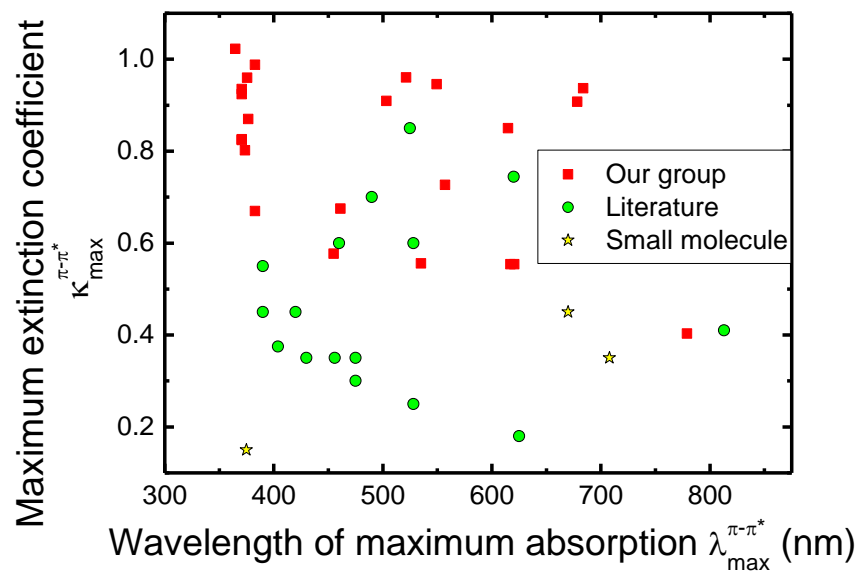

Figure 1: Extinction coefficient $\kappa$ (imaginary part of refractive index) spectra and maximum value of $\kappa$ for a range of conjugated polymers. (a) Spectra for a selection of conjugated polymers that have been widely studied for organic solar cells. The maximum $\kappa$ lies at around 1 , while the energetic breadth of the primary optical transition varies by $<20 \%$. (b) Extinction coefficient maximum, $\kappa_{\max }^{\pi-\pi^{*}}$, as a function of peak absorption wavelength for a larger set of materials, including fluorene, paraphenylene, thiophene, cyclopentadithiophene, carbazole, isoindigo, benzodithiophene and quinoxalene based polymers. The best performing solar cell materials have maximum extinction coefficients of approximately 1 . The specific materials and relevant references used to prepare this figure are tabulated in SI Section S4.1. 


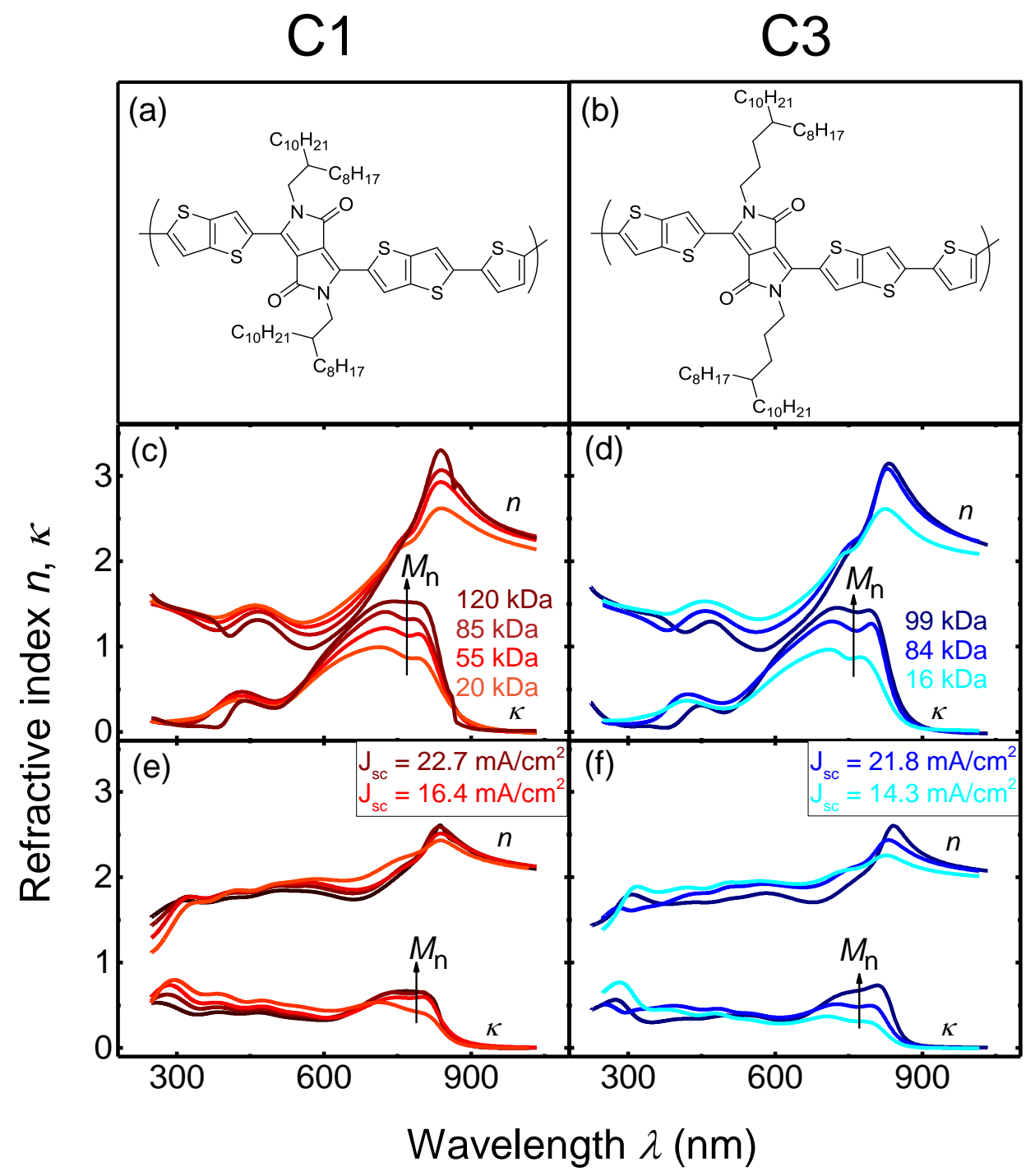

Figure 2: Molecular structures and refractive indices of DPP-TT-T C1 and C3 polymers. (a) and (b) are the chemical structures of C1 and C3 respectively (synthesis details are given in Ref. ${ }^{21}$ ), (c) and (d) show the refractive index data for pristine polymer films of C1 and C3 respectively, and (e) and (f) show the corresponding data for 1:2 polymer: $\mathrm{PC}_{70} \mathrm{BM}$ blend films of $\mathrm{C} 1$ and $\mathrm{C} 3$ respectively. The C1 polymers have number-average molecular weights of 20, 55, 85 and 120 $\mathrm{kDa}$; the C3 polymers have number-average molecular weights of 16, 84 and $99 \mathrm{kDa}$. See Tables S1.1 and S1.2 for full molecular weight information. Also included for reference is the shortcircuit current of devices made with high and low molecular weight C1 (120 kDa in dark red and 
$55 \mathrm{kDa}$ in red), and with high and low molecular weight C3 (84 kDa in dark blue and $16 \mathrm{kDa}$ in light blue). See SI Section S1 for full molecular weight information, and Section S2 for full J-V data. 
(a)

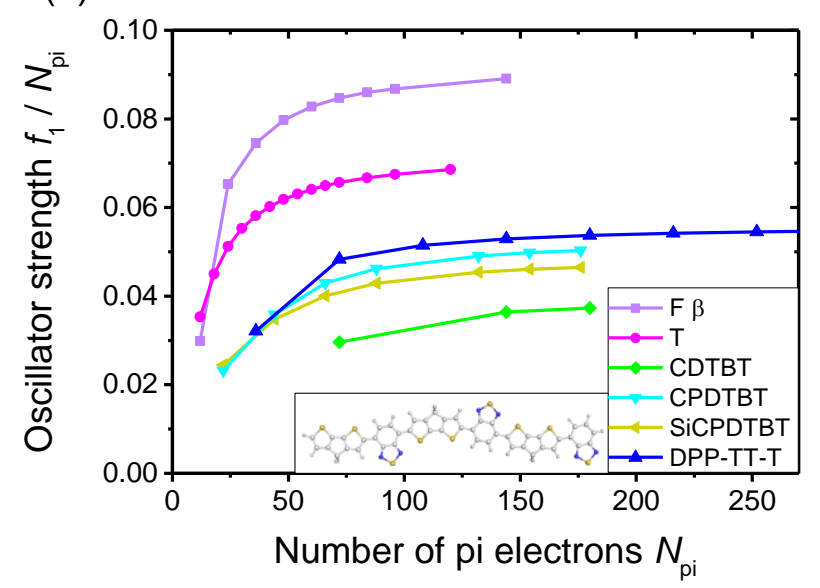

(b)

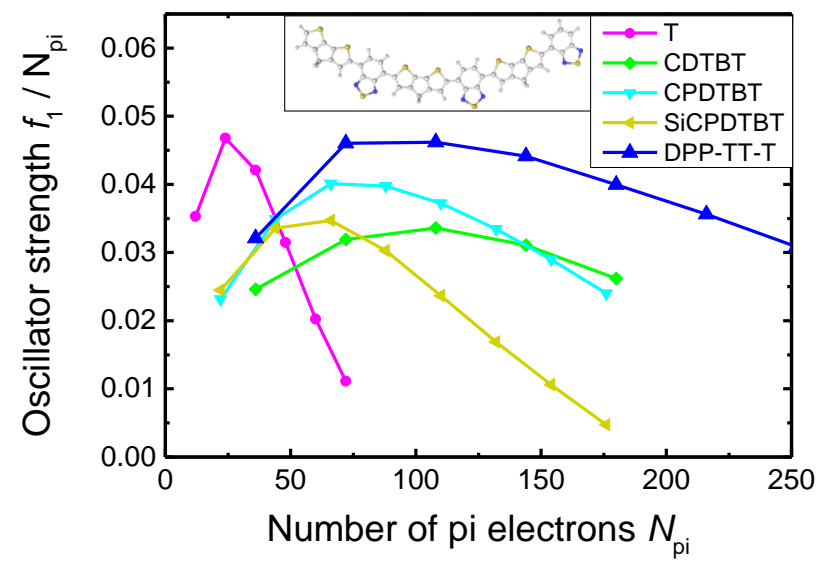

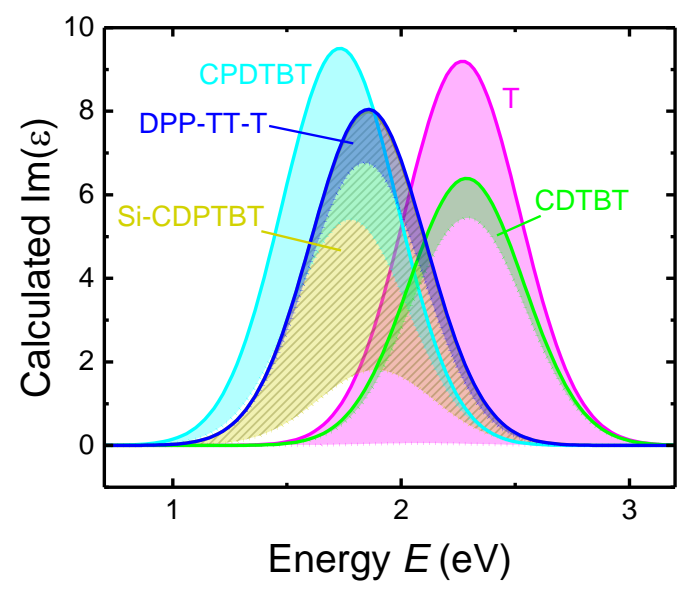

Figure 3: Calculated oscillator strength, normalized by the number of $\pi$-electrons $N_{\mathrm{pi}}$, and corresponding $\varepsilon_{2}$ spectra. (a) Normalised oscillator strength $f_{1}$ of the lowest energy transition as a function of number of pi electrons $N_{\mathrm{pi}}$, for oligomers of various structures in the alternating (trans) configuration, calculated using TDDFT with CAM-B3LYP/6-31g*. The inset shows the calculated all-trans conformation of a CPDTBT oligomer. (b) As (a) for cis configuration. The inset shows the calculated all-cis conformation of a CPDTBT oligomer. (c) Modelled $\varepsilon_{2}$ spectra calculated from Eq. 2 using calculated $f_{1}$ values for oligomers containing a similar number (around 140-160) of $\pi$ electrons. The transition is broadened with a Gaussian function of width $0.25 \mathrm{eV}$. The all-trans case is represented by solid lines, while the all-cis case is dotted lines. The shaded area represents the range of intermediate conformations which the polymer is expected to adopt. Note that oligofluorene does not planarise, and so the trans/cis representation does 
not apply. $\beta$-phase oligofluorene is planar so is included in (a), but it does not form the cis conformation so is omitted from (b) and (c).

(a)

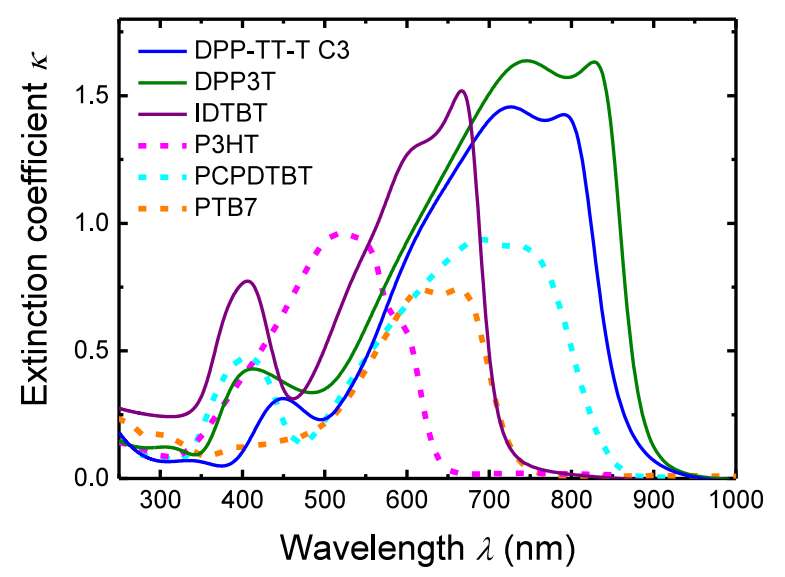

(b)

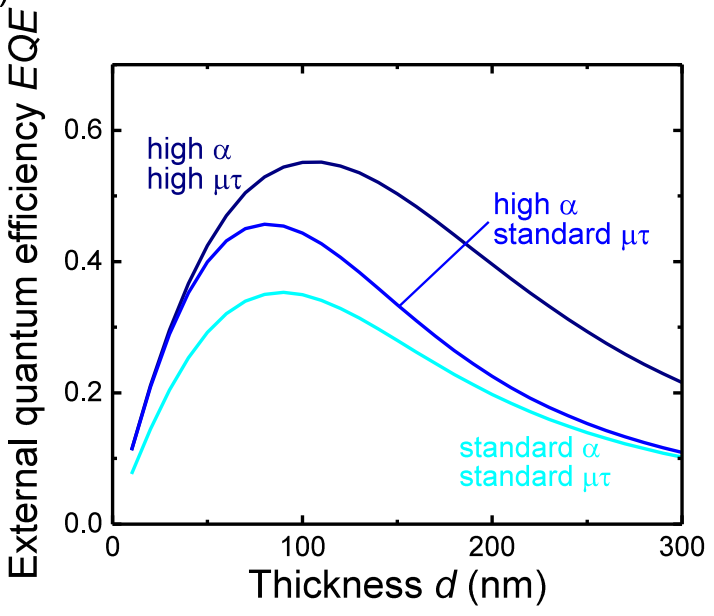

Figure 4: Extinction coefficient of polymers with high and low theoretical persistence length $\left(\lambda_{\mathrm{p}}\right)$, and the effect of absorption coefficient on external quantum efficiency $E Q E$. (a) Extinction coefficient of a range of conjugated polymers: the dashed lines represent polymers which exhibit low theoretical persistence length $\lambda_{\mathrm{p}}$ while DPP3T, DPP-TT-T C3 and IDTBT exhibit high $\lambda_{\mathrm{p}}$ and are represented by solid lines. The C3 polymer shown here has $M_{\mathrm{n}}=99 \mathrm{~kg} \mathrm{~mol}^{-1}$. The low $\lambda_{\mathrm{p}}$ polymers shown are P3HT, PCPDTBT and PTB7. (b) Simple model of photocurrent external quantum efficiency as a function of thickness, calculated assuming drift-dominated current and first order carrier recombination, for the case of standard (monochromatic) absorption and standard carrier collection ( $\alpha=1.6 \times 10^{5} \mathrm{~cm}^{-1}, \mu \tau=10^{-14} \mathrm{~m}^{2} \mathrm{~V}^{-1}$ ) (light blue line), enhanced absorption and standard carrier collection $\left(\alpha=2.4 \times 10^{5} \mathrm{~cm}^{-1}, \mu \tau=10^{-14} \mathrm{~m}^{2} \mathrm{~V}^{-1}\right)$ (medium blue), and enhanced absorption and enhanced carrier collection $\left(\alpha=2.4 \times 10^{5} \mathrm{~cm}^{-1}, \mu \tau=2 \times 10^{-14} \mathrm{~m}^{2} \mathrm{~V}^{-}\right.$ 1) (dark blue). Maximum EQE is determined by the strength of optical absorption and by the collection efficiency. See S10 for details. 


\section{Energy $(\mathrm{eV})$}

a)

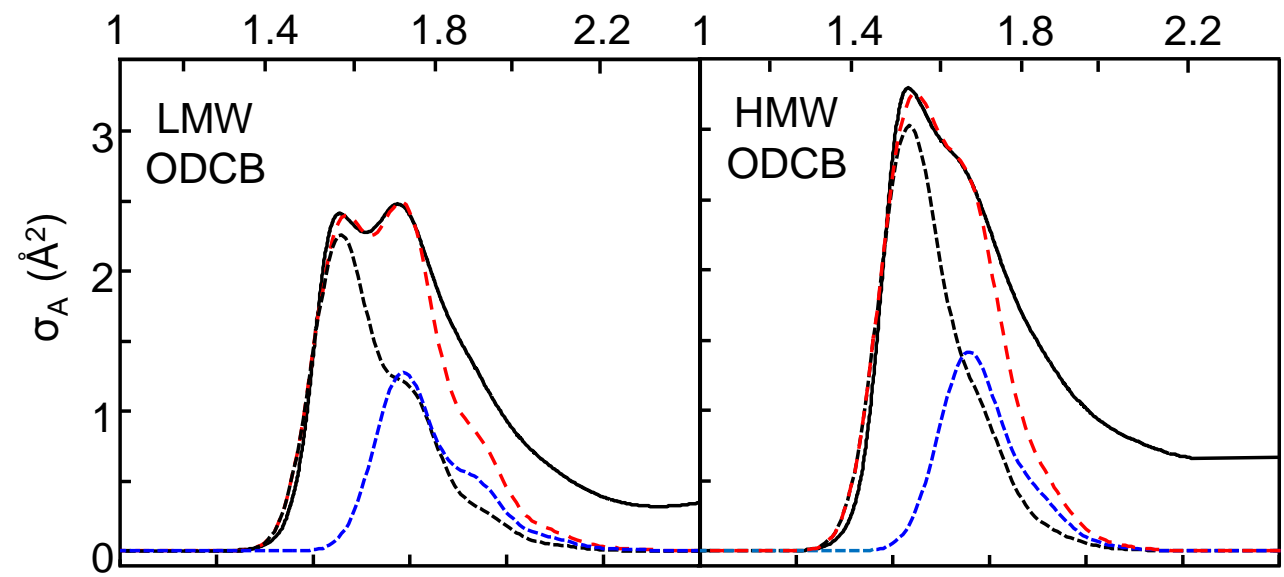

b)

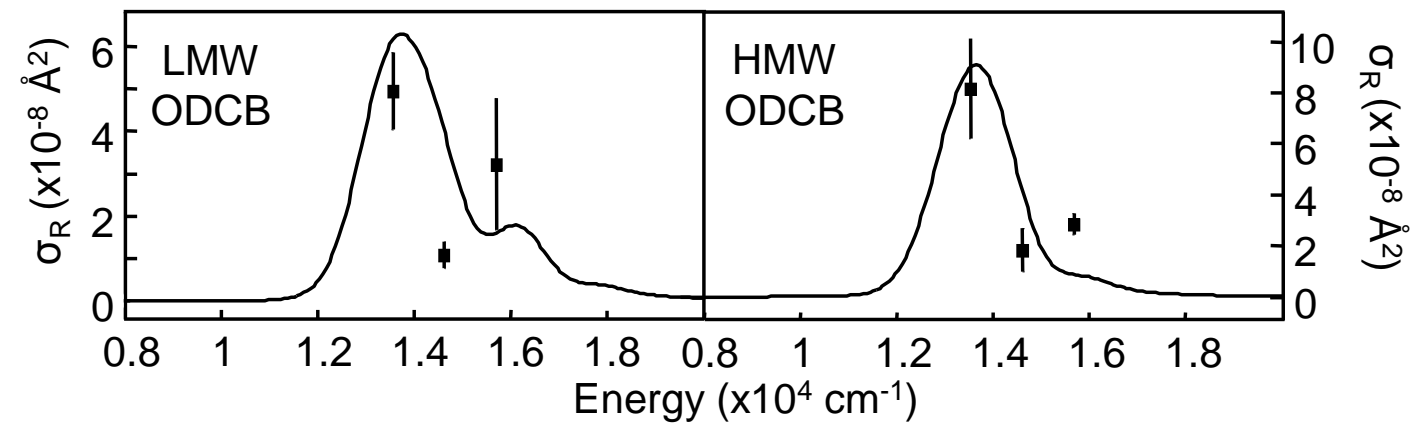

c)
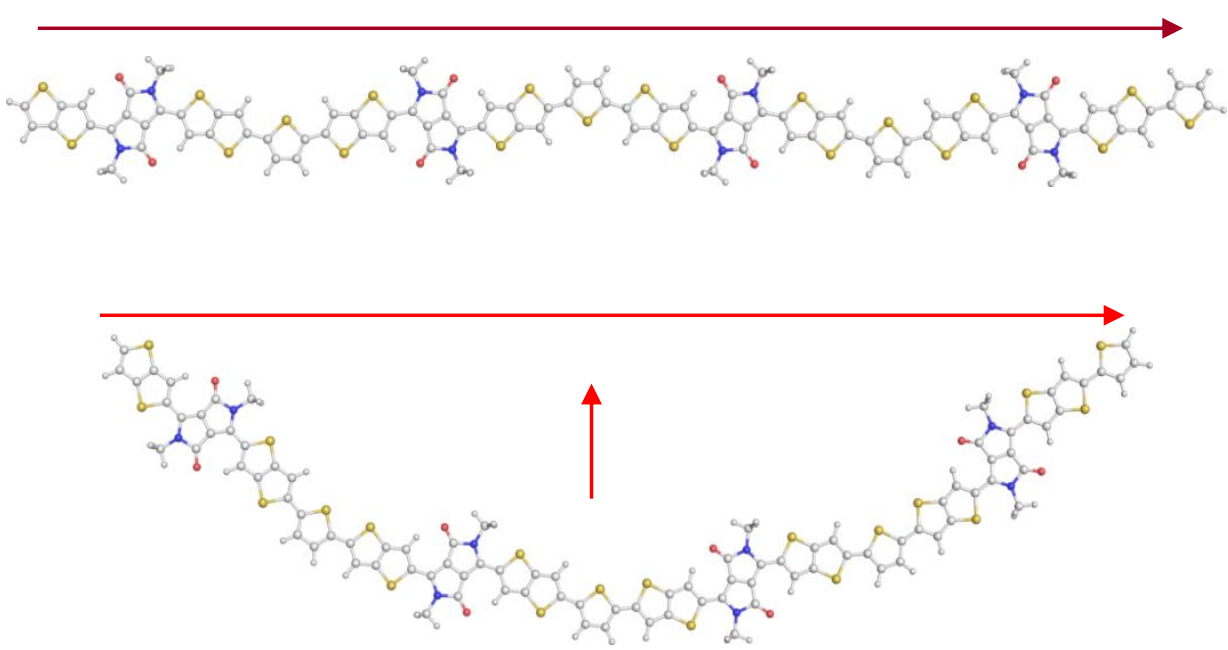

Figure 5: Resonance Raman Intensity Analysis (RRIA) of dilute solutions of high and low molecular weight C3 polymer in 1,2-dichlorobenzene. (a) Experimental (solid black line) and calculated (dashed red line) absorption cross sections of C3 polymers in 1,2-dichlorobenzene. The dashed black and blue lines represent the absorption spectra for the two transitions that contribute to the overall absorption band. (b) Corresponding experimental (points) and calculated (solid line) 
Raman excitation profiles for the $1492 \mathrm{~cm}^{-1}$ mode of LMW and HMW C3 polymer. The error bars are the standard deviation from 3 measurements of the absolute cross section of each mode at each excitation wavelength. (c) Schematic of the direction of transition dipole moments for first two electronic transitions in linear and curved tetramers of DPP-TT-T. The top oligomer is the all-trans conformation, whose first electronic transition dipole is directed along the oligomer backbone, while the second transition is dark and is thus not shown. The bottom oligomer is the all-cis conformation, whose first electronic transition dipole is directed along the backbone while the second is directed perpendicular to the backbone. The Raman spectra can be explained by a sum of contributions from linear and curved oligomers.

(a)

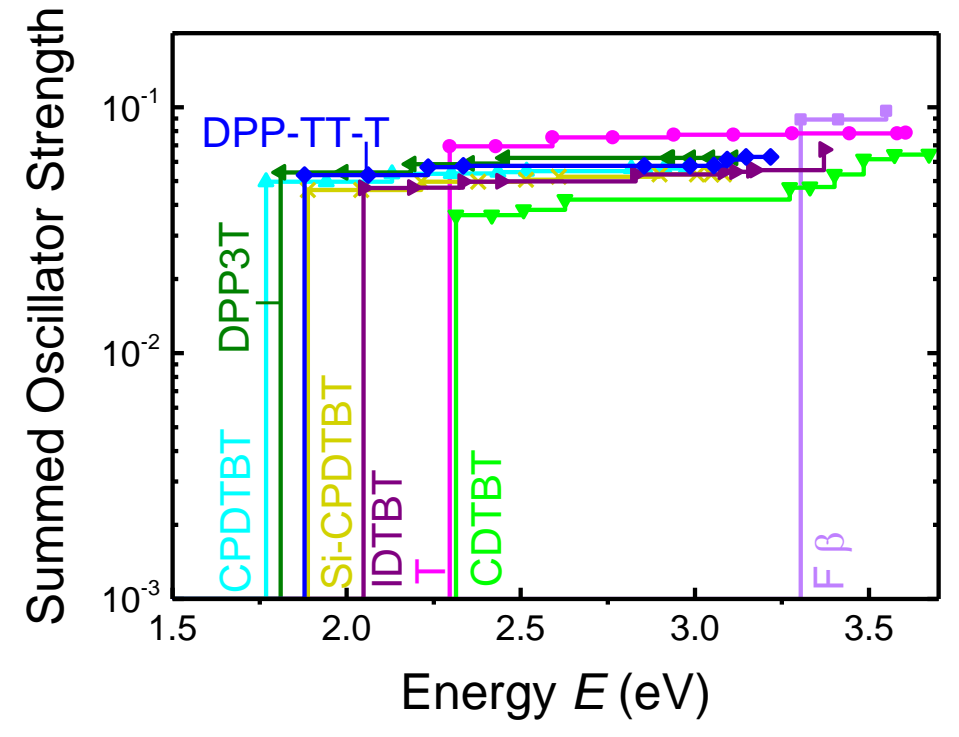


(b)

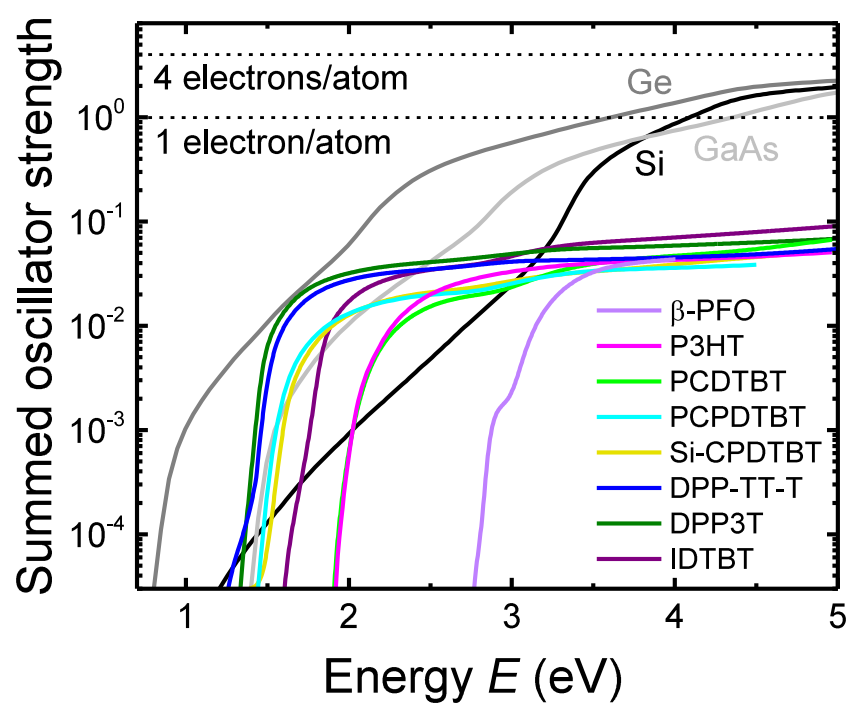

Figure 6 Calculated and experimental summed oscillator strength per $\pi$-system electron. (a)

Calculated summed oscillator strength per electron in the $\pi$-system at increasing photon energies for oligomers in the all-trans conformation with $120<\mathrm{N}_{\mathrm{pi}}<165$, using TDDFT with CAM-B3LYP/6-31g*. (b) Experimental summed oscillator strength per $\pi$-system electron for a range of polymers. The corresponding data for key inorganic photovoltaic materials ( $\mathrm{Si}$, Ge and GaAs) are shown for comparison.

\section{References}

1 Pope, M., Swenberg, C. E. \& Pope, M. Electronic processes in organic crystals and polymers. 2nd edn, (Oxford University Press, 1999).

2 Köhler, A. \& Bässler, H. Electronic Processes in Organic Semiconductors: An Introduction. (John Wiley \& Sons, 2015).

3 Spano, F. C. \& Silva, C. H- and J-aggregate behavior in polymeric semiconductors. Annual review of physical chemistry 65, 477-500 (2014).

4 Tian, B., Zerbi, G., Schenk, R. \& Mullen, K. Optical-Spectra and Structure of Oligomeric Models of Polyparaphenylenevinylene. Journal of Chemical Physics 95, 3191-3197 (1991).

5 Puschnig, P. et al. Electronic, optical, and structural properties of oligophenylene molecular crystals under high pressure: An ab initio investigation. Physical Review B 67 (2003).

6 Prest, W. M. \& Luca, D. J. Origin of the Optical Anisotropy of Solvent Cast Polymeric Films. Journal of Applied Physics 50, 6067-6071 (1979).

$7 \quad$ Prest, W. M. \& Luca, D. J. The Alignment of Polymers during the Solvent-Coating Process. Journal of Applied Physics 51, 5170-5174 (1980). 
Koynov, K. et al. Molecular weight dependence of chain orientation and optical constants of thin films of the conjugated polymer MEH-PPV. Macromolecules 39, 8692-8698 (2006).

9 Clark, J., Chang, J. F., Spano, F. C., Friend, R. H. \& Silva, C. Determining exciton bandwidth and film microstructure in polythiophene films using linear absorption spectroscopy. Applied Physics Letters 94 (2009).

10 Hestand, N. J. et al. Confirmation of the Origins of Panchromatic Spectra in Squaraine Thin Films Targeted for Organic Photovoltaic Devices. Journal of Physical Chemistry C 119, 18964-18974 (2015).

11 Yao, K., Xu, Y. X., Li, F., Wang, X. F. \& Zhou, L. A Simple and Universal Method to Increase Light Absorption in Ternary Blend Polymer Solar Cells Based on Ladder-Type Polymers. Adv Opt Mater 3, 321-327 (2015).

12 Sjoqvist, J., Linares, M., Lindgren, M. \& Norman, P. Molecular dynamics effects on luminescence properties of oligothiophene derivatives: a molecular mechanics-response theory study based on the CHARMM force field and density functional theory. Physical Chemistry Chemical Physics 13, 17532-17542 (2011).

13 Hedstrom, S., Henriksson, P., Wang, E., Andersson, M. R. \& Persson, P. Light-harvesting capabilities of low band gap donor-acceptor polymers. Physical Chemistry Chemical Physics 16, 24853-24865 (2014).

14 Grimm, B., Risko, C., Azoulay, J. D., Bredas, J. L. \& Bazan, G. C. Structural dependence of the optical properties of narrow bandgap semiconductors with orthogonal donoracceptor geometries. Chemical Science 4, 1807-1819 (2013).

15 Mishra, A. et al. A-D-A-type S, N-Heteropentacenes: Next-Generation Molecular Donor Materials for Efficient Vacuum-Processed Organic Solar Cells. Advanced materials 26, 7217-7223 (2014).

$16 \mathrm{Xu}, \mathrm{Y}$. X. et al. Improved Charge Transport and Absorption Coefficient in Indacenodithieno[3,2-b]thiophene-based Ladder-Type Polymer Leading to Highly Efficient Polymer Solar Cells. Advanced materials 24, 6356-6361 (2012).

17 Rau, U. Reciprocity relation between photovoltaic quantum efficiency and electroluminescent emission of solar cells. Physical Review B 76 (2007).

18 Campoy-Quiles, M., Alonso, M. I., Bradley, D. D. C. \& Richter, L. J. Advanced Ellipsometric Characterization of Conjugated Polymer Films. Advanced Functional Materials 24, 21162134 (2014).

19 Bronstein, H. et al. Thieno[3,2-b]thiophene-diketopyrrolopyrrole-containing polymers for high-performance organic field-effect transistors and organic photovoltaic devices. Journal of the American Chemical Society 133, 3272-3275 (2011).

20 Wood, S. et al. Natures of optical absorption transitions and excitation energy dependent photostability of diketopyrrolopyrrole (DPP)-based photovoltaic copolymers. Energy \& Environmental Science (2015).

21 Meager, I. et al. Photocurrent Enhancement from Diketopyrrolopyrrole Polymer Solar Cells through Alkyl-Chain Branching Point Manipulation. Journal of the American Chemical Society 135, 11537-11540 (2013).

22 Meager, I. et al. Power conversion efficiency enhancement in diketopyrrolopyrrole based solar cells through polymer fractionation. Journal of Materials Chemistry C 2, 8593-8598 (2014).

23 Kline, R. J. et al. Dependence of regioregular poly(3-hexylthiophene) film morphology and field-effect mobility on molecular weight. Macromolecules 38, 3312-3319 (2005).

24 Chang, J.-F. et al. Molecular-weight dependence of interchain polaron delocalization and exciton bandwidth in high-mobility conjugated polymers. Physical Review B 74 (2006).

25 Fox, M. Optical properties of solids. (Oxford University Press, 2001).

26 Koch, F. P. Synthesis and Physical Chemistry of a 'Monomer-up Approach' PhD thesis, ETH Zurich, (2013). 
27 Schumacher, S. et al. Effect of exciton self-trapping and molecular conformation on photophysical properties of oligofluorenes. The Journal of chemical physics 131, 154906 (2009).

28 Li, W. et al. One-Step Synthesis of Precursor Oligomers for Organic Photovoltaics - A Comparative Study between Polymers and Small Molecules. ACS applied materials \& interfaces (2015).

29 van Faassen, M., de Boeij, P. L., van Leeuwen, R., Berger, J. A. \& Snijders, J. G. Ultranonlocality in time-dependent current-density-functional theory: Application to conjugated polymers. Physical Review Letters 88 (2002).

30 Albuquerque, R. Q., Hofmann, C. C., Kohler, J. \& Kohler, A. Diffusion-Limited Energy Transfer in Blends of Oligofluorenes with an Anthracene Derivative. J Phys Chem B 115, 8063-8070 (2011).

31 Rossi, G., Chance, R. R. \& Silbey, R. Conformational Disorder in Conjugated Polymers. Journal of Chemical Physics 90, 7594-7601 (1989).

32 Soos, Z. G. \& Schweizer, K. S. Absorption-Spectrum of Flexible Conjugated Polymers - the Weak-Disorder Limit. Chemical Physics Letters 139, 196-200 (1987).

33 Flory, P. J. Statistical mechanics of chain molecules. (Interscience Publishers, 1969).

34 Jackson, N. E. et al. Conformational Order in Aggregates of Conjugated Polymers. Journal of the American Chemical Society 137, 6254-6262 (2015).

35 Chung, W. J., Shibaguchi, H., Terao, K., Fujiki, M. \& Naito, M. Evaluation of Global Conformation of Polydialkylsilane Using Correlation between Persistence Length and Excitonic Absorption. Macromolecules 44, 6568-6573 (2011).

36 Vanhee, S. et al. Synthesis and characterization of rigid rod poly(p-phenylenes). Macromolecules 29, 5136-5142 (1996).

37 Bronstein, H. et al. Indacenodithiophene-co-benzothiadiazole Copolymers for High Performance Solar Cells or Transistors via Alkyl Chain Optimization. Macromolecules 44, 6649-6652 (2011).

38 Venkateshvaran, D. et al. Approaching disorder-free transport in high-mobility conjugated polymers. Nature 515, 384-388 (2014).

39 Marcus, M., Tozer, O. R. \& Barford, W. Theory of optical transitions in conjugated polymers. II. Real systems. Journal of Chemical Physics 141 (2014).

40 Hestand, N. J. \& Spano, F. C. The Effect of Chain Bending on the Photophysical Properties of Conjugated Polymers. J Phys Chem B 118, 8352-8363 (2014).

41 Hayes, S. C. \& Silva, C. Analysis of the excited-state absorption spectral bandshape of oligofluorenes. The Journal of chemical physics 132, 214510 (2010).

42 Myers, A. B., Mathies, R. A. \& Spiro, T. Biological applications of Raman spectroscopy. Resonance Raman Spectra of Polyenes and Aromatics 2, 1 (1987).

43 van Franeker, J. J., Turbiez, M., Li, W. W., Wienk, M. M. \& Janssen, R. A. J. A real-time study of the benefits of co-solvents in polymer solar cell processing. Nature communications 6 (2015).

44 Campoy-Quiles, M., Nelson, J., Bradley, D. \& Etchegoin, P. Dimensionality of electronic excitations in organic semiconductors: A dielectric function approach. Physical Review $B$ 76 (2007). 


\section{Experimental Methods}

The different batches and fractions of $\mathrm{C} 1$ and $\mathrm{C} 3$ polymers used in this work are listed in Tables S1.1 and S1.2 respectively; corresponding gel permeation chromatography traces are displayed in Figure S1.1. The polymers were all made according to the synthesis described in Ref. ${ }^{21}$. DPPTT-T films and corresponding polymer: $\mathrm{PC}_{70} \mathrm{BM}(1: 2)$ blends for ellipsometry were blade coated using a Zehntner ZAA2300 blade coater at speeds ranging from $10 \mathrm{~mm} \mathrm{~s}^{-1}$ to $70 \mathrm{~mm} \mathrm{~s}^{-1}$ in order to vary film thickness in a range between $40 \mathrm{~nm}$ and $140 \mathrm{~nm}$. A multi-sample investigation was performed, which typically included three film thicknesses and two different substrates (spectrosil and silicon) for each material (5 to 6 samples). Additional measurements were performed on selected systems using two other substrates, glass/PEDOT:PSS (30nm) and Silicon/SiO2(1030nm). A total of 92 samples were measured across multiple batches of the three polymers. Variable-angle spectroscopic ellipsometry (VASE) and (in some cases, photometry) data for the samples described above were acquired at three to five angles of incidence between 55 and 75 degrees using a Sopralab GES-5E rotating polarizer spectroscopic ellipsometer (SEMILAB) coupled to a CCD detector. The data in Figure 1 were collected by the groups at Imperial and at ICMAB over a period between 2003 and 2015. These VASE data were collected using the following ellipsometers: Semilab (Sopra) GES5E, a RC2 instrument from J. A. Woollam Co., Inc. (USA) and a SOPRA GES-5 instrument. The standard critical point model $(\mathrm{SCP})^{44}$ was used to fit the data for both pristine DPP-TT-T films and corresponding blends.

Bulk heterojunction solar cells were fabricated with inverted (ITO/ZnO/active layer $/ \mathrm{MoO}_{3} / \mathrm{Ag}$ ) configuration and tested under simulated $100 \mathrm{~mW} \mathrm{~cm}{ }^{-2} \mathrm{AM} 1.5 \mathrm{G}$ illumination in air. ITO-coated glass substrates were purchased from Psiotec and sonicated in acetone and isopropanol before being subjected to an oxygen plasma treatment. The $\mathrm{ZnO}$ film was then prepared by the solgel method as reported in the literature. The active layers were in all cases a polymer: $\mathrm{PC}_{70} \mathrm{BM}$ blend ratio of $1: 2(\mathrm{w} / \mathrm{w})$. The active layer films were spincoated from $15 \mathrm{mgmL}^{-1}$ solutions in a chloroform:1,2-dichlorobenzene (4:1 v/v) solvent mixture. The $\mathrm{MoO}_{3}$ and Ag electrodes for all devices in this study were evaporated in one batch, and the device area was $4.5 \mathrm{~mm}^{2}$.

RRIA was performed on dilute solutions ( $\left.~ 5.5 \times 10^{-5} \mathrm{M}-6.5 \times 10^{-5} \mathrm{M}\right)$ of low and high molecular weight batches of DPP-TT-T C3 (C3-16 and C3-99 respectively), prepared in chloroform (HPLC, 99.9\%, Aldrich) and 1,2-dichlorobenzene (HPLC, 99.9\% Aldrich), which were used as received. The exact concentration of each DPP-TT-T solution was obtained from the UVVis spectrum (Shimadzu), using the pseudo molar extinction coefficient of DPP-TT-T at high molecular weight (C3-99) in chloroform at $823 \mathrm{~nm}\left(90 \times 10^{3} \mathrm{~L} \mathrm{~mol}^{-1} \mathrm{~cm}^{-1}\right)$ and at $811 \mathrm{~nm}$ in 1,2- 
dichlorobenzene $\left(85 \times 10^{3} \mathrm{~L} \mathrm{~mol}^{-1} \mathrm{~cm}^{-1}\right)$. The pseudo molar extinction coefficient of low molecular weight DPP-TT-T (C3-16) in chloroform was $70 \times 10^{3} \mathrm{~L} \mathrm{~mol}^{-1} \mathrm{~cm}^{-1}$ at $807 \mathrm{~nm}$ and $65 \times 10^{3} \mathrm{~L} \mathrm{~mol}^{-1}$ $\mathrm{cm}^{-1}$ at $796 \mathrm{~nm}$ in 1,2-dichlorobenzene.

The absolute resonance Raman (RR) intensities of DPP-TT-T for the two molecular weights dissolved either in chloroform or 1,2-dichlorobenzene were measured with excitation at $636.8,682.9$ and $737.8 \mathrm{~nm}$. The 682.9 and $636.8 \mathrm{~nm}$ excitation wavelengths employed in the RR experiments were produced via Raman shifting the second and third harmonics from a Qswitched Nd:YAG laser (PRO-230, $30 \mathrm{~Hz}$, Spectra Physics) at 532 and $355 \mathrm{~nm}$, respectively, in a homemade $1 \mathrm{~m}$ stainless-steel tube containing $\mathrm{H}_{2}$ gas at 1.5 bar. The $737.8 \mathrm{~nm}$ excitation wavelength was produced via Raman shifting the fundamental from a Q-switched Nd:YAG laser (Lab-190, $30 \mathrm{~Hz}$, Spectra Physics) at $1064 \mathrm{~nm}$, in a $1 \mathrm{~m}$ stainless-steel tube containing $\mathrm{H}_{2}$ gas at 40 bar (Light Age). The excitation light was focused into a spinning cell consisting of an EPR suprasil tube (diameter: $4 \mathrm{~mm}$ ) attached to a rheostat-controlled motor for choice of rotation speed. Use of the spinning cell prolonged the lifetime of the samples. Modest excitation energies ( $\sim 15 \mu$ per pulse) were employed to avoid decomposition of the sample. The Raman scattered light was collected in a backscattering geometry and delivered to a $0.75 \mathrm{~m}$ focal-length Czerny-Turner spectrograph, equipped with a 1200-grooves/mm holographic grating. The slit width was set to $100 \mu \mathrm{m}$ providing for $2 \mathrm{~cm}^{-1}$ spectral resolution at the wavelengths used in this work. The scattered light was detected by a thermoelectrically-cooled $1340 \times 400$ pixel, backilluminated deep depletion CCD detector (PIXIS-XB:400 BR, Princeton Instruments). Each spectrum presented here is the accumulation of 12-24 10 min spectra. Frequency calibration of the spectra was accomplished with the use of toluene. In the calculation of the absolute RR cross sections the $1002 \mathrm{~cm}^{-1}$ mode of toluene (9.4 M) was used as an external standard. 